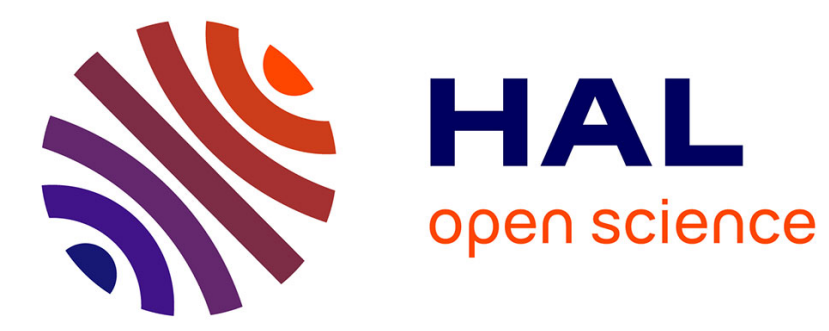

\title{
Linear MMSE detection technique for MC-CDMA
}

Jean-François Hélard, Jean-Yves Baudais, Jacques Citerne

\section{To cite this version:}

Jean-François Hélard, Jean-Yves Baudais, Jacques Citerne. Linear MMSE detection technique for MC-CDMA. Electronics Letters, 2000, 36 (7), pp.665-666. hal-00283455

\section{HAL Id: hal-00283455 \\ https://hal.science/hal-00283455}

Submitted on 18 Sep 2014

HAL is a multi-disciplinary open access archive for the deposit and dissemination of scientific research documents, whether they are published or not. The documents may come from teaching and research institutions in France or abroad, or from public or private research centers.
L'archive ouverte pluridisciplinaire HAL, est destinée au dépôt et à la diffusion de documents scientifiques de niveau recherche, publiés ou non, émanant des établissements d'enseignement et de recherche français ou étrangers, des laboratoires publics ou privés. 


\title{
A novel linear MMSE detection technique for MC-CDMA
}

\author{
Jean-François Hélard, Jean-Yves Baudais, Jacques Citerne \\ Institut National des Sciences Appliquées \\ LCST, 20, Avenue des Buttes de Coësmes, 35043 Rennes Cedex \\ email : jean-yves.baudais@insa-rennes.fr
}

\begin{abstract}
A novel detection technique for MC-CDMA systems based on the MMSE criterion applied per user is presented. Simulation results in a Rayleigh fading channel show very good performance, mainly for non full load systems.
\end{abstract}

Introduction : Since 1993, Multi-Carrier Code Division Multiple Access (MC-CDMA) has been the subject of much research, and appears to be a very good candidate to support multimedia services in mobile radio communications [1]. The MC-CDMA transmitter spreads in the frequency domain the original data stream over different subcarriers using a given spreading code. For a synchronous system as the downlink mobile radio communication channel, the application of orthogonal codes such as Walsh-Hadamard codes guarantees the absence of Multiple Access Interference (MAI) in a Gaussian channel. However, through a frequency selective fading channel, all the subcarriers have different amplitude levels and different phase shifts, which results in a loss of the orthogonality among users and then generates MAI. So, after direct FFT and frequency deinterleaving, the received sequence must be "equalized" by using one tap adaptive equalizer per subcarrier to make up for the phase and amplitude distortions caused by the mobile radio channel. To combat the MAI, various basic detection techniques such as Maximum Ratio Combining (MRC), Equal Gain Combining (EGC), Orthogonal Restoring Combining (ORC) or Minimum Mean Square Error (MMSE) may be used. This last technique, based on the MMSE criterion applied independently on each subcarrier [2] achieves better performance. This letter describes a novel detection technique based on the MMSE criterion applied per user which provides performance improvements, mainly for non full load systems.

System description : The block diagram of the considered MC-CDMA transmitter and receiver is depicted in Fig. 1. Each data symbol $x^{n}{ }_{j}$ assigned to user $j, j=1, \ldots, N_{u}$ and transmitted during the symbol interval $n$ is multiplied with its user specific Walsh-Hadamard spreading code $\boldsymbol{C}_{j}=\left[c_{j}^{l}, c_{j}^{2}, \ldots, c_{j}^{L c}\right]^{T}$ of length $L_{c}$, where $[.]^{T}$ denotes matrix transposition. $L_{C}$ corresponds to the bandwidth expansion factor and is equal to the maximum number of simultaneous active users. The $j^{\text {th }}$ column vector of the $L_{C} \times L_{C}$ matrix $\boldsymbol{C}$ corresponds to the spreading code $\boldsymbol{C}_{j}$ of the user $j$. The vector of the data symbols transmitted during the $n^{\text {th }}$ OFDM symbol by all the users can be written $\boldsymbol{X}^{n}=\left[x_{1}^{n}, x_{2}^{n}, \ldots, x_{j}^{n}, \ldots, x_{L c}^{n}\right]^{T}$, with $x_{j}^{n}=0$ when user $j$ is inactive.

Since we consider the synchronous downlink of an MC-CDMA system, the different data modulated spreading codes of the $N_{u}$ users can be added before Serial-to-Parallel (S/P) conversion. Furthermore, the $N_{u}$ user signals are supposed to be transmitted with the same power. The number $N_{p}$ of subcarriers which are 4PSK modulated is chosen equal to the spreading code length $L_{C}$. For this study, frequency non-selective Rayleigh fading per subcarrier and time invariance during one OFDM symbol are assumed. Besides, the absence of Intersymbol Interference and Intercarrier Interference is guaranteed by the use of a guard interval longer than the delay spread of the impulse response of the channel. Under theses assumptions and considering a frequency interleaving, the complex channel fading coefficients are independent for each subcarrier and can be estimated for the subcarrier $k$ by $h_{k}=\rho_{k} e^{i \theta k}$. The signal received after the inverse OFDM operation (serial to parallel conversion and direct FFT) and deinterleaving, can be expressed as : 


$$
\boldsymbol{R}=\left[r_{1}, r_{2}, \ldots, r_{N p}\right]^{T}=\boldsymbol{H} . \boldsymbol{C} . \boldsymbol{X}+\boldsymbol{N}
$$

where the $N_{p}$ x $N_{p}$ diagonal matrix $\boldsymbol{H}=\operatorname{diag}\left\{h_{1}, \ldots, h_{N p}\right\}$ describes the complex channel frequency response and $N=\left[n_{1}, n_{2}, \ldots, n_{N p}\right]^{T}$ is the vector containing the AWGN terms with $n_{k}$ representing the noise term at the subcarrier $k$ with variance given by $\sigma_{N}^{2}=E\left\{\left|n_{k}\right|^{2}\right\}, k=1, \ldots, N_{p}$.

Basic detection techniques : After equalization the received signal can be written :

$$
\boldsymbol{Y}=\left[y_{1}, y_{2}, \ldots, y_{N_{p}}\right]^{T}=\boldsymbol{G} \cdot \boldsymbol{R}=\boldsymbol{G} . \boldsymbol{H} \cdot \boldsymbol{C} \cdot \boldsymbol{X}+\boldsymbol{G} \cdot \boldsymbol{N}
$$

The $N_{p} \mathrm{x} N_{p}$ matrix $\boldsymbol{G}$ represents the complex equalization coefficients obtained from the channel estimation which can be based on known transmitted pilot symbols inserted between the data carriers. Various basic detection techniques can be implemented : Maximum ratio combining (MRC), Equal Gain Combining (EGC), Orthogonality Restoring Combining (ORC)...Among all those well-known single-user detection techniques, MMSE (Minimum Mean Square Error) equalization offers the best results. It minimizes the mean square value of the error $\varepsilon_{k}$ between the signal $s_{k}$ transmitted on subcarrier $k$ and the assigned output $y_{k}$ of the equalizer. The equalization coefficients based on this MMSE criterion applied independently per carrier are equal to :

$$
g_{k}=\frac{h_{k}^{*}}{\left|h_{k}\right|^{2}+\frac{1}{\gamma_{c}}}=\frac{h_{k}^{*}}{\left|h_{k}\right|^{2}+\frac{L_{c}}{N_{u}} \cdot \frac{1}{\gamma_{X}}}
$$

where $\gamma_{C}$ is the subcarrier signal to noise ratio, $\gamma_{X}$ is the signal to noise ratio of the received data symbol $x$ and (.)* denotes complex conjugation. For all these basic detection techniques, the matrix $\boldsymbol{G}$ is diagonal : the received sequence is then equalized by using a bank of $N_{p}$ adaptive one tap equalizers. It results from this that the complexity of the equalizer is low. However, this MMSE equalization per carrier method is not optimal, since it does not take into account the despreading process and thus does not minimize the mean square error at the input of the threshold detector T.

MMSE equalization per user : The aim of the proposed method is to minimize the mean square error between the transmitted symbol $x_{j}$ and the estimated one $\hat{x}_{j}$. Let $\boldsymbol{W j}=\left[w_{j}^{0}, w_{j}^{l}, \ldots, w_{j}^{N p}\right]$ be the optimal weighting vector. The estimated symbol of the $j^{\text {th }}$ user can be written:

$$
\hat{x}_{j}=\boldsymbol{W}_{j}^{T} \cdot \boldsymbol{R}=\boldsymbol{C}_{j}^{\boldsymbol{T}} \cdot \boldsymbol{G} \cdot \boldsymbol{R}
$$

According to the Wiener filtering, the optimal weighting vector is equal to:

$$
\boldsymbol{W}_{j}=\Gamma_{R, R}^{-1} \cdot \Gamma_{R, x_{j}}
$$

where $\Gamma_{R, R}$ is the autocorrelation matrix of the received vector $\boldsymbol{R}$ and $\Gamma_{R, x j}$ is the cross-correlation vector between the desired symbol $x_{j}$ and the received signal vector $\boldsymbol{R}$. Those terms are equal to :

$$
\begin{aligned}
& \Gamma_{R, R}=E\left\{\boldsymbol{R}^{*} \cdot \boldsymbol{R}^{T}\right\}=\boldsymbol{H}^{*} \cdot \boldsymbol{C} \cdot E\left\{X^{*} \cdot \boldsymbol{X}^{T}\right\} \cdot \boldsymbol{C}^{T} \cdot \boldsymbol{H}+E\left\{N^{*} \cdot N^{T}\right\} \\
& \Gamma_{\boldsymbol{R}, x_{j}}=E\left\{\boldsymbol{R}^{*} \cdot x_{j}\right\}=\boldsymbol{H}^{*} \cdot \boldsymbol{C} \cdot E\left\{X^{*} \cdot x_{j}\right\}
\end{aligned}
$$

Then, the optimal weighting vector can be written:

$$
\boldsymbol{W}_{j}^{T}=E\left\{x_{j} \cdot \boldsymbol{X}^{* T}\right\} \cdot \boldsymbol{C}^{T} \boldsymbol{H}^{*} \cdot\left(\boldsymbol{H} \cdot \boldsymbol{C} \cdot E\left\{\boldsymbol{X} \cdot \boldsymbol{X}^{* T}\right\} \cdot \boldsymbol{C}^{T} \cdot \boldsymbol{H}^{*}+E\left\{\boldsymbol{N} \cdot \boldsymbol{N}^{* T}\right\}\right)^{-1}
$$

The subcarrier noises have the same variance and are independent. Thus, $E\left\{N \cdot N^{* T}\right\}=\sigma_{N}^{2} . I$ where $I$ is the identity matrix. Since the user signals have the same power $\left(E\left\{x_{j}^{2}\right\}=E_{s}\right)$ and are independant, we can write $E\left\{\boldsymbol{X} . \boldsymbol{X}^{* T}\right\}=E_{s} \cdot \boldsymbol{A}$, where $\boldsymbol{A}=\left\{a_{i j}\right\}$ is a diagonal matrix with the term $a_{j j}=1$ if the user $j$ is active and $a_{j j}=0$ if the user $j$ is inactive. Then, the equalization coefficient matrix is : 


$$
\boldsymbol{G}=\boldsymbol{H}^{*} \cdot\left(\boldsymbol{H} \cdot \boldsymbol{C} \cdot \boldsymbol{A} \cdot \boldsymbol{C}^{\boldsymbol{T}} \cdot \boldsymbol{H}^{*}+\frac{\sigma_{N}^{2}}{E_{s}} . \boldsymbol{I}\right)^{-1}
$$

In the full load case $\left(N_{u}=L_{c}\right)$ and only in that case, the quantity $\boldsymbol{C} . \boldsymbol{A} \cdot \boldsymbol{C}^{T}$ is equal to the identity matrix and the equalization coefficients matrix $\boldsymbol{G}$ is a diagonal matrix with the $k^{\text {th }}$ subcarrier equalization coefficient equal to the former expression (3).

Simulation results : In Fig. 2, the performance of various detection systems with $L_{c}=N_{p}=64$ is presented, taking into account the number $N_{u}$ of active users versus the necessary $\mathrm{E}_{\mathrm{b}} / \mathrm{N}_{0}$ to achieve a $\mathrm{BER}=10^{-3}$. A full load system is obtained for $N_{u}=L_{c}=N_{p}=64$. The loss due to the guard interval, the synchronisation and the channel estimation is not taken into account. In any case, EGC and MRC perform poorly. The performance of the MMSE per carrier system with the equalization coefficients optimised independently on each subcarrier according to the expression (3) is compared to the performance of the MMSE per user system with the equalization coefficient matrix $\boldsymbol{G}$ equal to the expression (8). For full load systems $\left(N_{u}=64\right)$, the performance of the two MMSE approaches are the same, while for non full load systems the MMSE per user criterion achieves a gain of more than $2 \mathrm{~dB}$ with $N_{u}=32$ or 16 .

With the aim of improving the performance of the receiver still further, Multi-user Detection (MD) techniques such as Interference Cancellation can be implemented. Parallel Interference Cancellation (PIC) detectors estimate the interference due to the simultaneous other users in order to remove this multiple user interference component from the received signal. Interference Cancellation can be carried out iteratively in multiple detection stages. A performance comparison of two stage parallel interference cancellation systems with the two MMSE techniques versus the number of active users (with $\mathrm{E}_{\mathrm{b}} / \mathrm{N}_{0}=8 \mathrm{~dB}$ ) is presented in Fig. 3 with $L_{c}=N_{p}=64$. In any case, the new MMSE per user detection scheme outperforms the MMSE per carrier system. For example, with $E_{b} / N_{0}=8 d B$ and for a BER $=10^{-3}$, the base station can supply $N_{u}=21$ users with PIC-MMSE per carrier and up to $N_{u}=31$ users with PIC-MMSE per user detection.

Conclusions : Taking advantage of the despreading process, this novel approach based on the MMSE criterion applied per user and no more per carrier offers very good results in the case of non full load systems with linear detection but also with multi-user PIC detection schemes.

\section{References :}

[1] S. Hara, R. Prasad, "Overview of multicarrier CDMA", IEEE Communications Magazine, December 1997, pp 126-133.

[2] S. Kaiser, "Analytical performance evaluation of OFDM-CDMA mobile radio systems", Proceedings First European Personal and Mobile Communications Conference (EPMCC'95), Bologna, Italy, November 1995, pp 215-220. 


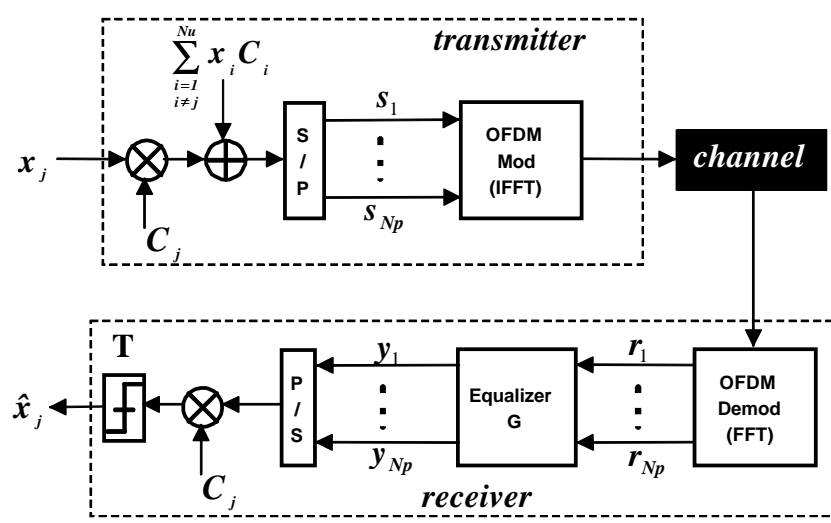

Fig. 1 MC-CDMA transmitter and receiver

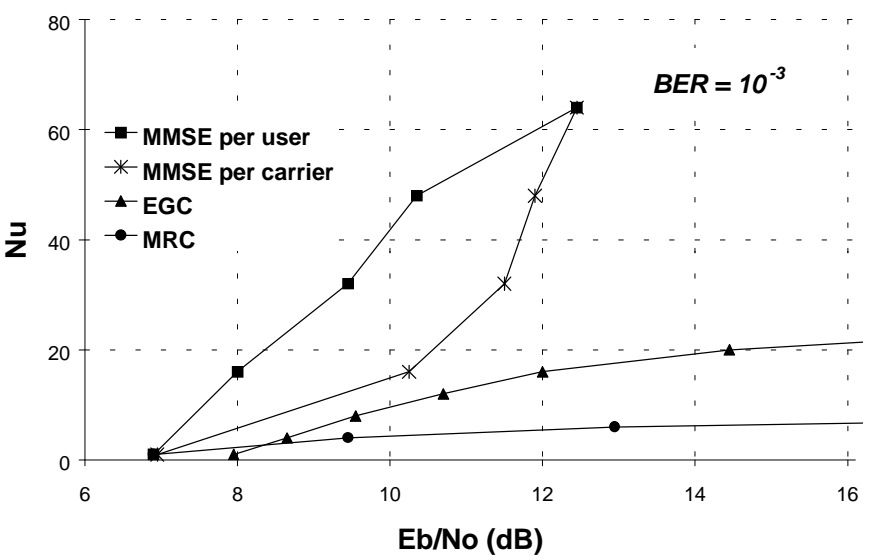

Fig. 2 Maximum number $N_{u}$ of active users versus Eb/NO for a $B E R=10^{-3} ; N_{p}=L_{c}=64$

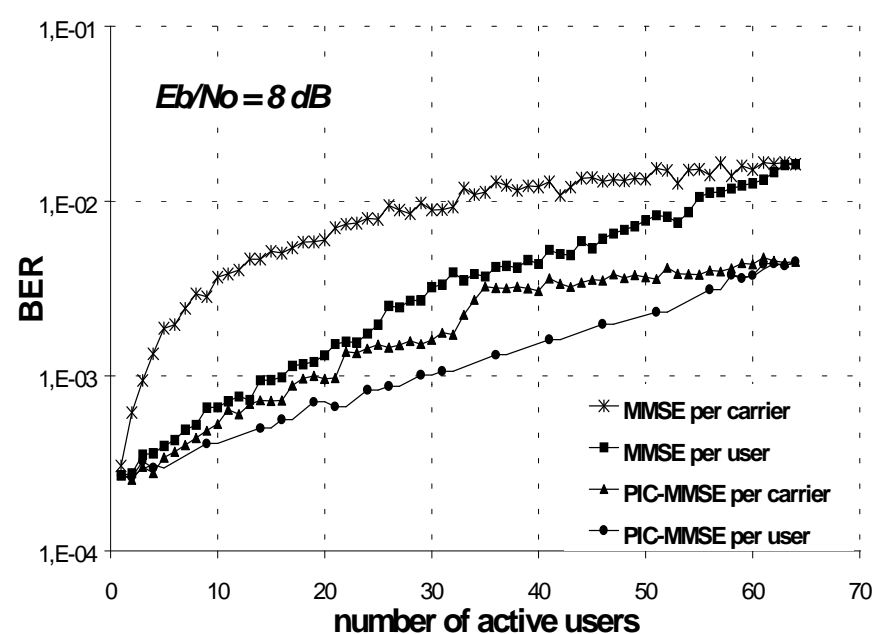

Fig. 3. MMSE detection performance versus the number $N_{u}$ of active users for $\mathrm{Eb} / \mathrm{NO}=8 d \mathrm{~B} ; N_{p}=L_{c}=64$ 